\title{
National Norms and Correlates of the PHQ-8 and GAD-7 in Parents of School-age Children
}

\author{
Stefanie L. Sequeira $\mathbb{D}^{1} \cdot$ Kayley E. Morrow ${ }^{1} \cdot$ Jennifer S. Silk ${ }^{1} \cdot$ David J. Kolko $^{2} \cdot$ Paul A. Pilkonis $^{2} \cdot$ Oliver Lindhiem $^{2}$
}

Accepted: 17 June 2021 / Published online: 2 July 2021

(C) The Author(s), under exclusive licence to Springer Science+Business Media, LLC, part of Springer Nature 2021

\begin{abstract}
Anxiety and depressive disorders are global public health concerns, and research suggests that these disorders are common in parents and can adversely influence family functioning. However, little is known about normative levels of anxiety and depressive symptoms in parents of school-age children. The present study reports on generalized anxiety and depressive symptoms in 1570 parents and guardians of a nationally representative sample of children ages five to twelve years using two widely used and validated questionnaires: the eight-item variant of the Patient Health Questionnaire depression scale (PHQ-8) and the seven-item Generalized Anxiety Disorder scale (GAD-7). Moderate to severe levels of generalized anxiety symptoms were reported in $12.7 \%$ of the total sample and moderate to severe levels of depressive symptoms were reported in $14.1 \%$ of the sample; $17.7 \%$ of the sample reported moderate to severe levels of either generalized anxiety or depressive symptoms. This percentage was higher for females, younger parents and guardians, and parents and guardians reporting lower household incomes. These data, collected online in early 2018, may be useful for researchers and clinicians studying and treating anxiety and depression in parents. Further, these data provide a baseline for researchers currently studying the impact of changes related to the novel coronavirus (COVID-19) pandemic (e.g., school closures) on the mental health of parents of school-age children.
\end{abstract}

Keywords Anxiety $\cdot$ Generalized anxiety $\cdot$ Depression $\cdot$ Parents $\cdot$ National sample

\section{Highlights}

- Almost $18 \%$ of parents and guardians of school-age children in the United States report moderate to severe symptoms of generalized anxiety or depression.

- Symptoms of generalized anxiety and depression are higher in younger parents and guardians and those reporting the lowest household incomes.

- Normative levels of generalized anxiety and depressive symptoms are provided for researchers, clinicians, and other health professionals studying and treating parents of school-age children.

Anxiety and depressive symptoms and disorders are common in adulthood and associated with significant impairment in social, physical, and vocational domains, as well as increased risk for future psychiatric diagnoses, substance

Stefanie L. Sequeira

SLS234@pitt.edu

1 Department of Psychology, University of Pittsburgh, Pittsburgh, PA, USA

2 Department of Psychiatry, University of Pittsburgh, Pittsburgh, PA, USA abuse, and suicidal behaviors (Kessler et al., 2012; Löwe et al., 2008; Pine et al., 1998; Rucci et al., 2003; Cuijpers et al., 2013; Haller et al., 2014). These disorders may be highly distressing for parents of young children, who already face many stressors, and the symptoms can adversely influence functioning in the family system. A breadth of research has examined how anxiety and depression in parents might affect parenting behaviors (e.g., Teetsel et al., 2014; England et al., 2009; Lovejoy et al., 2000) and children's socioemotional health (e.g., Barket et al. 2011; Goodman et al., 2011; Garber \& Cole, 2010; Lawrence et al., 2019). For example, anxiety disorders have been linked to increases in paternal controlling behaviors 
and increases in maternal reinforcement of children's dependence in anxiety-provoking situations (Teetsel et al., 2014). Though these parenting behaviors may be intended to increase security and comfort for the child, they may unintentionally foster higher anxiety in the child, particularly for children at genetic risk for anxiety. Depressive symptoms, such as decreased motivation or low energy, in parents could also contribute to increased parent-child conflict (Kane \& Garber, 2004) or more hostile, negative, and withdrawn parenting (England et al., 2009), potentially related to the effects of depressive symptoms on parents' cognitive, interpersonal, and/or physical functioning.

Though we know that symptoms of generalized anxiety and depression may be highly distressing for parents and contribute to altered functioning in various domains, we do not yet know how common these symptoms are in U.S. parents; research on normative levels of self-reported generalized anxiety symptoms and depressive symptoms in the general population of U.S. parents is lacking. This information is critical for researchers, clinicians, and other health professionals who use self-report measures, such as the Generalized Anxiety Disorder seven-item scale (GAD-7; Spitzer et al., 2006) and the eight-item variant of the Patient Health Questionnaire depression scale (PHQ-8; Kroenke et al., 2001; 2009), to assess generalized anxiety and depressive symptoms in parents and to guide recommendations for treatment. Normative levels of generalized anxiety symptoms and depressive symptoms are likely to be higher in parents relative to the general adult population, especially considering limited prior evidence of heightened rates of major depressive disorder (MDD) and generalized anxiety disorder (GAD) in parents. Though most nationallyrepresentative U.S. studies of MDD and GAD prevalence do not note parental status specifically, some nationally representative studies of GAD and MDD rates in parents of children under age 18 do exist (e.g., England \& Sim, 2009; Ertel et al., 2011; Lieb et al., 2002). For example, in a subsample of over 2,000 U.S. adults with at least one child under the age of 18 years from the National Comorbidity Survey Replication (NCS-R) in 2002, 12-month prevalence of MDD was $7.2 \%$ (England \& Sim, 2009), slightly higher than the 12 -month prevalence of $6.7 \%$ for the total sample of U.S. adults in 2002 (Kessler et al., 2005). Significantly higher rates of past-year MDD were found in women $(10.0 \%)$ than men $(4.3 \%)$ (England \& Sim, 2009). Similarly, in a separate nationally representative study of almost 9,000 U.S. mothers living with a child under age $18,10.2 \%$ of mothers were diagnosed with MDD over the past year (around 2001-2002), which was higher than the rate of MDD reported in the full sample of women from this database (6.9\%) (Ertel et al., 2011).

High levels of anxiety and depressive symptoms in parents of young children have also been found outside the
U.S. For example, in a study of 913 families in Norway, $8-14 \%$ of mothers of at least one child $4.5-12.5$ years of age reported clinically significant symptoms of both anxiety and depression measured using the Hopkins Symptom Checklist (Skipstein et al., 2010), while in almost 2,000 mothers of at least one 3-year-old in Canada, $12.5 \%$ reported clinically significant symptoms of depression, measured using the Center for Epidemiologic Studies Depression Scale (CES-D; Mughal et al., 2018). Additionally, in the EDEN mother-child study based in France, mean levels of CES-D depressive symptoms increased in mothers from four months postpartum to three and five years following the child's birth (van der Waerden et al., 2015). While females are about twice as likely to experience major depression relative to males (Bromet et al., 2011), around $4 \%$ of fathers of 21-month-olds from the Avon Longitudinal Study of Parents and Children reported clinically significant symptoms of depression on the Edinburgh Postnatal Depression Scale (Ramchandani et al., 2008), similar to MDD rates seen for fathers in the NCS-R subsample. Depressive symptoms in parents of young children are thus a global concern.

Importantly, while some nationally representative studies of rates of GAD and MDD in U.S. parents exist (e.g., England \& Sim, 2009; Ertel et al., 2011), to our knowledge, no studies have focused specifically on identifying normative levels of generalized anxiety symptoms and depressive symptoms in parents living in the U.S. To address this gap in the literature, we conducted secondary analysis of data collected in early 2018. Using a large sample of parents and guardians of at least one child age 5 to 12 years, we examined levels of generalized anxiety and depressive symptoms using two common measures: The GAD-7 (Spitzer et al., 2006) and the PHQ-8 (Kroenke et al., 2001, 2009). These measures are brief, reliable, and valid, and are administered easily in a wide range of settings, including online (e.g., Erbe et al., 2016; van Ballegooijen et al., 2016) and in primary care offices. It has been estimated that almost half of primary care patients with depression are identified by their physician (Simon et al., 1999), emphasizing the importance of these screening questionnaires for ascertaining anxiety and depression in adults. Though normative data for the GAD-7 and PHQ-8 exist for the general U.S. population of adults (Spitzer et al., 2006; Kroenke et al., 2009), normative symptom data for U. S. parents of school-age children specifically have not previously been published.

In addition to examining normative levels of generalized anxiety and depressive symptoms, we examined sociodemographic correlates of these symptoms in the present study. Limited information has been gathered on risk factors for generalized anxiety symptoms and depressive symptoms in U.S. parents of school-age children, though more is 
known about risk factors for GAD and MDD in parents and in the general population of U.S. adults. Identifying correlates of anxiety and depressive symptoms is of critical clinical importance because many parents report symptoms of generalized anxiety and depression that are distressing and affect individual and family functioning without meeting diagnostic criteria for GAD or MDD. Understanding risk factors for GAD and MDD may, however, guide hypotheses regarding risk factors for generalized anxiety and depressive symptoms examined more continuously. In parents specifically, findings from nationally representative studies suggest that prevalence rates of MDD do not differ by the age of the child (England \& Sim, 2009) or the number of children in the home (Ertel et al., 2011). However, higher rates of MDD have been found in parents who were divorced, separated, or widowed, relative to parents who were never married, married, or cohabitating (England \& Sim, 2009). In parents and in the general population of adults, a large body of literature also shows that females report higher levels of depressive symptoms and have higher rates of MDD and GAD than males (Vesga-López et al., 2008; England \& Sim, 2009; Kessler et al., 2012; Salk et al., 2017). In addition, large-scale population studies have found that younger adults report higher depressive severity (Xu et al., 2017) and are at greater risk for MDD (Hasin et al., 2018), while middle age (i.e., 30-44) is associated with highest risk for GAD (Grant et al., 2005, 2009). Research in U.S. mothers specifically has shown higher rates of MDD in younger women (Ertel et al., 2011). Though poverty status was not associated with depression in parents in the 2002 NCS-R data, lower income has been linked to higher rates of GAD and MDD in the general population of U.S. adults (e.g., Kessler et al., 2003; Grant et al., 2005; Hasin et al., 2018) and in U.S. mothers specifically (Ertel et al., 2011).

In the NCS-R subsample of parents, 12-month prevalence of major depression did not significantly differ by race or ethnicity; however, differences in lifetime prevalence were found, such that the highest rates of MDD were seen in Black immigrants from the Caribbean (29.7\%), and higher rates were seen in non-Hispanic white parents (19.6\%) relative to African American parents (12.0\%) and Asian parents (8.8\%). In U.S. mothers specifically, research has found lower rates of MDD in Black and Hispanic mothers relative to non-Hispanic white mothers and Native American mothers (Ertel et al., 2011). Higher rates of MDD and GAD in non-Hispanic white adults relative to Black, Hispanic, and/or Asian adults have also been reported in the general U.S. adult population (Riolo et al., 2005; Grant et al., 2005; Breslau et al., 2005; Himle et al., 2009; Asnaani et al., 2010; Hasin et al., 2018). It should be noted that while lower rates of MDD or GAD in Black, Hispanic, and Asian adults could be related to a host of cultural and contextual factors, differences could also be related to biases in assessment (e.g., Perreira et al., 2005; Crockett et al., 2005) and diagnosis (Bailey et al., 2019).

The overall aim of the present study is descriptive; we provide normative levels of generalized anxiety and depressive symptoms using the GAD-7 and PHQ-8, respectively, in U.S. parents and guardians of school-age children. These data provide needed benchmarks for health professionals, clinicians, and researchers who use these common and well-validated questionnaires to assess anxiety and depressive symptoms in parents. Further, these data provide a baseline measure of average generalized anxiety and depressive symptoms in parents of young children prior to the coronavirus disease (COVID-19) pandemic, which may be critical for researchers studying the impact of the pandemic on anxiety and depressive symptoms in parents. We also provide data on sociodemographic correlates of generalized anxiety and depressive symptoms in this sample of parents and guardians. Based on previous large-scale studies on MDD and GAD correlates (e.g., England \& Sim, 2009; Ertel et al., 2011), we hypothesize that females, younger parents, parents who are divorced, separated, or widowed, and parents with lower incomes will report higher symptoms of depression and generalized anxiety. As research on differences in GAD or MDD by race or ethnicity in parents is inconsistent (e.g., England \& Sim, 2009), and research on differences by number of children in the home is limited (e.g., Ertel et al., 2011), we include these descriptive variables (i.e., race/ethnicity, number of children in the home) in the present report but do not make a priori hypotheses regarding associations between these variables and symptoms of generalized anxiety or depression.

\section{Methods}

\section{Participants}

A total of 1570 parents and guardians (63.7\% female; mean age $=41.5$ years, $\mathrm{SD}=10.6$ ) of a nationally representative sample of school-age children between the ages of 5 and 12 years were recruited using an internet panel from all 50 states and the District of Columbia in early 2018. Though some participants were legal guardians, this sample will be referred to solely as parents throughout the remainder of the paper for brevity. Parents were originally recruited to test a novel parenting questionnaire (Lindhiem et al., 2019). Demographic information can be found in Table 1 (also see Lindhiem et al., 2019; Sequeira, Silk, Woods, Kolko, \& Lindhiem, 2019). We collapse across U. S. region (i.e., South, West, Northeast, Midwest) for the present study, as participants in different regions did not significantly differ in parent age, gender, or household income $(p s>.05)$ and differences in rates of depression by 
Table 1 Demographic information with group differences in mean GAD-7 and PHQ-8 scores

\begin{tabular}{|c|c|c|c|c|c|c|c|}
\hline & \multirow[t]{2}{*}{$N(\%)$} & \multicolumn{2}{|c|}{ Mean (SD) } & \multicolumn{2}{|c|}{ Group Difference $^{* * *}$} & \multicolumn{2}{|c|}{ Cohen's $d$ or $f$ (effect size) } \\
\hline & & GAD7 & PHQ8 & GAD7 & PHQ8 & GAD7 & PHQ8 \\
\hline Gender & & & & $p=.004$ & $p=.011$ & $d=.15$ & $d=.13$ \\
\hline Female & $1000(63.7)$ & $3.7(5.1)$ & $4.2(5.4)$ & & & & \\
\hline Male & $570(36.3)$ & $3.0(4.3)$ & $3.5(4.9)$ & & & & \\
\hline Age Group (years) & & & & $p<.001$ & $p<.001$ & $f=.20$ & $f=.19$ \\
\hline $20-30^{\mathrm{a}}$ & $144(9.2)$ & $5.0(5.5)$ & $5.7(6.2)$ & $\mathrm{a}>\mathrm{c}, \mathrm{d}, \mathrm{e}$ & $\mathrm{a}>\mathrm{c}, \mathrm{d}, \mathrm{e}$ & & \\
\hline $31-40^{\mathrm{b}}$ & $704(44.8)$ & $4.0(5.2)$ & $4.5(5.5)$ & $\mathrm{b}>\mathrm{c}, \mathrm{d}, \mathrm{e}$ & $\mathrm{b}>\mathrm{c}, \mathrm{d}, \mathrm{e}$ & & \\
\hline $41-50^{\mathrm{c}}$ & $398(25.4)$ & $2.9(4.3)$ & $3.5(5.0)$ & $\mathrm{c}<\mathrm{a}, \mathrm{b} ; \mathrm{c}>\mathrm{e}$ & $c<a, b ; c>e$ & & \\
\hline $51-60^{\mathrm{d}}$ & $214(13.6)$ & $2.7(4.3)$ & $3.0(4.4)$ & $\mathrm{d}<\mathrm{a}, \mathrm{b}$ & $\mathrm{d}<\mathrm{a}, \mathrm{b}$ & & \\
\hline $61-82^{\mathrm{e}}$ & $110(7.0)$ & $1.4(2.3)$ & $1.9(2.7)$ & $\mathrm{e}<\mathrm{a}, \mathrm{b}, \mathrm{c}$ & $\mathrm{e}<\mathrm{a}, \mathrm{b}, \mathrm{c}$ & & \\
\hline Marital Status & & & & $p=.009$ & $p=.004$ & $f=.07$ & $f=.08$ \\
\hline Married/civil partnership ${ }^{\mathrm{a}}$ & $1118(71.2)$ & $3.2(4.6)$ & $3.7(5.0)$ & $\mathrm{a}<\mathrm{c}$ & $\mathrm{a}<\mathrm{c}$ & & \\
\hline Divorced/separated/ widowed ${ }^{\mathrm{b}}$ & $209(13.3)$ & $3.6(5.0)$ & $4.4(5.6)$ & & & & \\
\hline Never married ${ }^{c}$ & $243(15.5)$ & $4.3(5.5)$ & $4.8(5.7)$ & & & & \\
\hline Total Household Income & & & & $p<.001$ & $p<.001$ & $f=.15$ & $f=.19$ \\
\hline$\$ 0-\$ 29,999^{\mathrm{a}}$ & $372(23.7)$ & $4.5(5.7)$ & $5.5(5.9)$ & $a>c, d$ & $\mathrm{a}>\mathrm{b}, \mathrm{c}, \mathrm{d}$ & & \\
\hline$\$ 30,000-\$ 59,999^{b}$ & $418(26.6)$ & $3.7(4.9)$ & $4.1(5.5)$ & $b>d$ & $\mathrm{~b}<\mathrm{a} ; \mathrm{b}>\mathrm{d}$ & & \\
\hline$\$ 60,000-\$ 89,999^{c}$ & $304(19.4)$ & $2.7(3.9)$ & $3.4(4.7)$ & $\mathrm{c}<\mathrm{a}$ & $\mathrm{c}<\mathrm{a}$ & & \\
\hline$\$ 90,000+{ }^{\mathrm{d}}$ & $341(21.7)$ & $2.8(4.2)$ & $2.9(4.1)$ & $\mathrm{d}<\mathrm{a}, \mathrm{b}$ & $\mathrm{d}<\mathrm{a}, \mathrm{b}$ & & \\
\hline \multicolumn{8}{|l|}{ Race/Ethnicity } \\
\hline White & $1236(78.7)$ & $3.6(4.8)$ & $4.2(5.3)$ & $p=.391 *$ & $p=.067 *$ & $d=.07$ & $d=.14$ \\
\hline Black or African American & $218(13.9)$ & $3.5(5.4)$ & $3.8(5.2)$ & & & & \\
\hline Asian & $54(3.4)$ & $1.9(3.5)$ & $2.2(3.8)$ & & & & \\
\hline American Indian or Alaskan Native & $41(2.6)$ & $3.3(5.0)$ & $4.3(5.9)$ & & & & \\
\hline Native Hawaiian or Pacific Islander & $9(0.6)$ & $2.2(2.7)$ & $3.7(4.8)$ & & & & \\
\hline Other Race & $86(5.5)$ & $2.8(4.7)$ & $3.5(5.6)$ & & & & \\
\hline Hispanic, Latino, or Spanish Origin & $255(16.3)$ & $3.1(5.0)$ & $3.8(5.6)$ & $p=.256^{* *}$ & $p=.616^{* *}$ & $d=.08$ & $d=.04$ \\
\hline Number of Children in the Home & & & & $p=.198$ & $p=.557$ & $f=.06$ & $f=.03$ \\
\hline $1-2$ & $815(51.9)$ & $3.5(4.9)$ & $4.0(5.1)$ & & & & \\
\hline $3-4$ & $530(33.8)$ & $3.2(4.7)$ & $3.8(5.3)$ & & & & \\
\hline $5-17$ & $223(14.2)$ & $3.8(5.1)$ & $4.2(5.4)$ & & & & \\
\hline
\end{tabular}

The GAD-7 measures symptoms of generalized anxiety and the PHQ-8 measures symptoms of depression

*Difference between white-identifying parents and Black- or African American-identifying parents

**Difference between parents reporting and not reporting Hispanic, Latino, or Spanish origin

***Results from a Welch's t-test (2 groups) or one-way ANOVA with Bonferroni post-hoc tests (more than 2 groups)

U.S. region are not supported (Kessler et al., 2003; Hasin et al., 2018). The majority of participants were currently married (68.2\%); $3.0 \%$ were in civil or domestic partnerships, $15.5 \%$ were never married, $9.2 \%$ were divorced, $2.1 \%$ were separated, $2.0 \%$ were widowed. The majority of parents $(63.3 \%)$ reported having one child between the ages of 5 and $12,27.5 \%$ reported two children between ages 5 and 12 , and $9.2 \%$ reported three or more (maximum seven) children between ages 5 and 12 . Parents also reported on the total number of children in the home (see Table 1).

Parents reported on their race and ethnicity (see Table 1); 95.9\% identified as one race, $78.7 \%$ identified as White,
13.9\% identified as Black or African American, 3.4\% identified as Asian, and $16.3 \%$ identified as Hispanic, Latino, or Spanish origin. Racial and ethnic identities in this sample were similar to reports published by the U.S. Census Bureau in 2010, in which $97.1 \%$ of responders identified as one race, $72.4 \%$ identified as White, $12.6 \%$ identified as Black or African American, 4.8\% identified as Asian, and $16.3 \%$ identified as Hispanic or Latino. Total household income was also reported using a scale of 1-11 with intervals of $\$ 10,000$ (e.g., $1=\$ 0-10,000,11=\$ 100,000$ + ). Median household income was \$50,000-59,999, which was similar to the median household income of $\$ 63,179$ 
reported by the U.S. Census Bureau in 2018. For analytic purposes, four income groups based on quartiles in the data were created (see Table 1); two groups were below the median income for 2018 (\$0-\$29,999 and \$30,000$\$ 59,999)$ and two groups were above the median $(\$ 60,000$ $\$ 89,999$ and $\$ 90,000+$ ). A total of 135 participants preferred not to report on income.

\section{Procedure}

Data were collected online in January-February 2018. The GAD-7 (Spitzer et al., 2006) and PHQ-8 (Kroenke \& Spitzer, 2002) were among several questionnaires administered to participants using YouGov (www.yougov.com), a survey company that maintains a panel of over 1.2 million U.S. residents available for online surveys (see Lindhiem et al., 2019 for more details about the accrual and procedures for the panel). Participants included on the YouGov panels are recruited through online advertising, e-mail campaigns, partner contracts, random digit dialing, and mail (based on voter registration). Links to the survey were emailed to the panelists who opted-in and agreed to be contacted for relevant surveys. Participants could complete the surveys on any device with a web browser (e.g., phone, laptop, tablet). Research suggests that internet samples reasonably represent the overall population (Hays et al., 2015). Recent research from the Pew Research Center (2019) shows that $90 \%$ of Americans use the internet; this percentage is even higher in Americans ages 18-49, who made up the majority of the present sample. U.S. adults over the age of 65 are less likely to use the internet, which is unlikely to affect sampling for the present study, and adults with less than a high school degree are also less likely to use the internet (Anderson et al., 2019), which may present sampling bias for the present study.

The response rate to the emailed survey link was $52.8 \%$. Of those who initiated the survey, $46.2 \%$ met eligibility criteria (at least one child aged 5-12 years). Respondents were matched to a sampling frame of U.S. parents/guardians of children ages 5-12 years on key variables including gender, race/ethnicity, and age. This sampling frame was constructed using stratified sampling from the 2016 American Community Survey 1-year estimates conducted by the U.S. Census. The matched cases were weighted to the sampling frame using propensity scores.

\section{Measures}

\section{GAD-7}

The GAD-7 (Spitzer et al., 2006) is a seven-item questionnaire that asks participants how often they were bothered by certain symptoms over the past two weeks. The scale was developed using the Diagnostic and Statistical Manual of Mental Disorders, Fourth Edition (DSM-IV; American Psychiatric Association, 1994) symptom criteria for GAD and items from existing anxiety scales (Spitzer et al., 2006). Response options are "not at all," "several days," "more than half the days," and "nearly every day," which are scored on a 0-3 scale for a maximum score of 21 . A GAD-7 score of $\geq 10$ has an $89 \%$ sensitivity and $82 \%$ specificity for generalized anxiety disorder and has been shown to represent clinically significant anxiety (Spitzer et al., 2006). Reliability for the GAD-7 was high in the present sample (Cronbach's $\alpha=.91$ ). The American version of the GAD-7 is freely available (Spitzer et al., 2006).

\section{PHQ-8}

The PHQ-8 (Kroenke et al., 2001, 2009) is an eight-item questionnaire that asks participants how often they were bothered by certain symptoms over the past two weeks. Response options are "not at all," "several days," "more than half the days," and "nearly every day," which are scored on a $0-3$ scale for a maximum score of 24 . The PHQ-8 consists of eight of the nine symptoms on which the DSM-5 (and DSM-IV) diagnosis of major depressive disorder is based (American Psychiatric Association, 1994, 2013); the ninth question in the DSM-5 assesses suicidal or self-injurious thoughts, which was not included in this version of the PHQ because the research staff could not provide adequate intervention if required. Research suggests that omitting this last item has a small effect on scoring because this item is rarely endorsed in the general population (Huang et al., 2006; Kroenke \& Spitzer, 2002; Lee et al., 2007). A PHQ-8 score of $\geq 10$ has a $100 \%$ sensitivity rate and $95 \%$ specificity rate for major depression and has been shown to represent clinically significant depression (Kroenke et al., 2009). Reliability for the PHQ-8 was high in the present sample (Cronbach's $\alpha=.91$ ). The PHQ-8 questionnaire is also freely available online (Kroenke et al., 2009).

\section{Data Analysis}

Analyses were primarily descriptive. Means, standard deviations, and percentile scores for each measure were computed. We also examined differences in mean scores based on age, gender, race/ethnicity, total family income, marital status, and total number of children in the household. One-way ANOVAs with Bonferroni post-hoc tests for multiple comparisons were used to examine group differences in GAD-7 and PHQ-8 scores for most variables, with the exception of gender and race/ethnicity, for which Welch's $t$-tests were used. We examined differences in GAD-7 and PHQ-8 scores between parents identifying as 
White and parents identifying as Black or African American; other racial groups were too small in number to make meaningful comparisons. We also examined differences between parents endorsing versus not endorsing Hispanic, Latino, or Spanish origin. We computed Cohen's $d$ measures of effect sizes (for Welch's $t$-tests) or Cohen's $f$ measures of effect sizes (for one-way ANOVAs). For Cohen's $d$, an effect size of .2 is considered small, .5 is considered medium, and .8 is considered large (Cohen, 1988). For Cohen's $f$, an effect size of .1 is considered small, .25 is considered medium, and .5 is considered large (Cohen, 1988). We also examined the number and percentage of parents with GAD-7 and PHQ-8 scores at or above 10 , which may indicate clinically significant levels of anxiety and depression. We computed these values for the entire sample, as well as separately by gender, age, and income group.

Correlations, chi-square tests, or analyses of variance (ANOVAs) were also computed to examine associations between demographic variables (i.e., gender, age, income, marital status, race and ethnicity, and total number of children in the home). The type of test used differed depending on the variable type. Pearson's or Spearman's rho correlations $\left(r_{\mathrm{s}}\right)$ were used to examine associations between two continuous variables or a continuous and interval variable, respectively. Chi-square tests were used to examine differences between two nominal variables. Oneway ANOVAs or $t$-tests (with corrections for unequal variance when appropriate) were used to test differences between continuous or interval and nominal or dichotomous variables. Age and total number of children were entered continuously, gender and ethnicity were dichotomous variables, income was entered using the 1-11 interval scale, and race and marital status were nominal variables.

\section{Results}

\section{Preliminary Associations between Demographic Variables}

Parent income differed significantly by parent gender $(t(1433)=10.53, p<.001)$ and age $\left(r_{\mathrm{s}}=.22, p<.001\right)$; age also differed by gender $(t(1116)=10.63, p<.001)$ and was associated with total number of children in the home $(r=$ $-.16, p<.001)$. Female parents were younger than male parents and overrepresented in the lowest income groups. Older parents reported higher household incomes and fewer children in the home. Differences by racial group were found for gender $\left(\chi^{2}(6, N=1569)=25, p<.001\right)$ and income $(F(6,35)=12.27, p<.001)$; the majority of parents identifying as Black or African American were female (77\%) and $42 \%$ reported household incomes below
$\$ 30,000$. Marital status differed significantly $(p s<.005)$ by all variables. Of note, a higher percentage of female parents $(19.6 \%)$ than male parents $(8.2 \%)$ were never married. Additionally, divorced, widowed, or separated parents were older on average ( $M=47.8$ years) than never married parents $(M=37.4$ years $)$ and parents currently married or in civil/domestic partnerships $(M=41.9$ years). Finally, $54.7 \%$ of never married parents were in the lowest income group (\$0-\$29,999), compared to $16.5 \%$ of parents who were married or in civil or domestic partnerships.

\section{Descriptive Data}

Scores on the GAD-7 and PHQ-8 were highly correlated $(r=.77, p<.001)$. Scores on the GAD-7 ranged from 0 to $21(M=3.4, S D=4.8)$; scores on the PHQ-8 ranged from 0 to $24(M=4.0, S D=5.2)$. Table 1 includes mean GAD-7 and PHQ-8 scores by parent age, gender, marital status, income, race/ethnicity, and total number of children in the home. Means are also depicted visually in Figs. 1 and 2 (for age and gender) and Fig. 3 (for income group). Tables 2 and 3 provide percentiles for the GAD-7 and PHQ- 8 . Percentiles can be used to compare an individual's GAD-7 and PHQ-8 score with those found in the population reference group (i.e., parents of at least one 5- to- 12-year-old). For example, a GAD-7 score of 5 in a male parent indicates a percentile rank of $75 \%$ in the general population of parents and of $79 \%$ in a group of parents of the same gender. Similarly, a PHQ-8 score of 9 in a female parent indicates a percentile rank of $85 \%$ in the general population of parents and of $83 \%$ in a group of parents of the same gender.

\section{Group Differences in Mean GAD-7 and PHQ-8 Scores}

Significant differences in GAD-7 and PHQ-8 scores were found for parent gender, age, marital status, and income groups, but not for race/ethnicity or number of children at

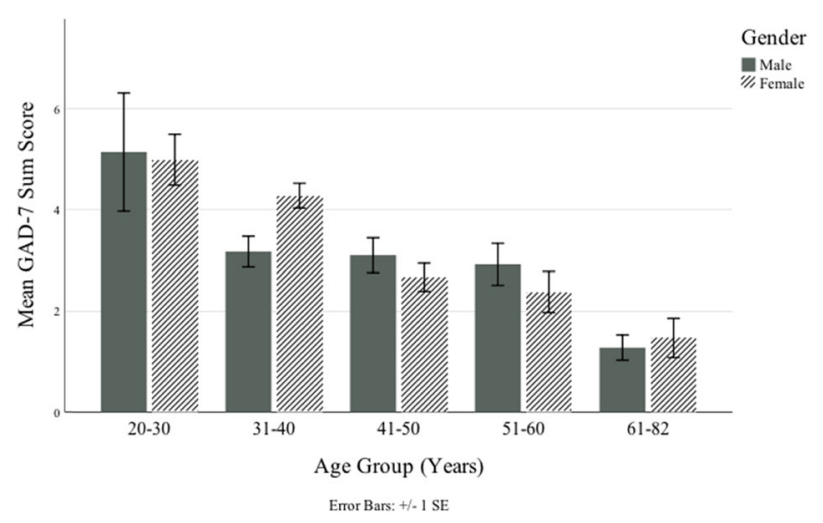

Fig. 1 Differences in mean GAD-7 scores (raw scores) by parent age and gender 


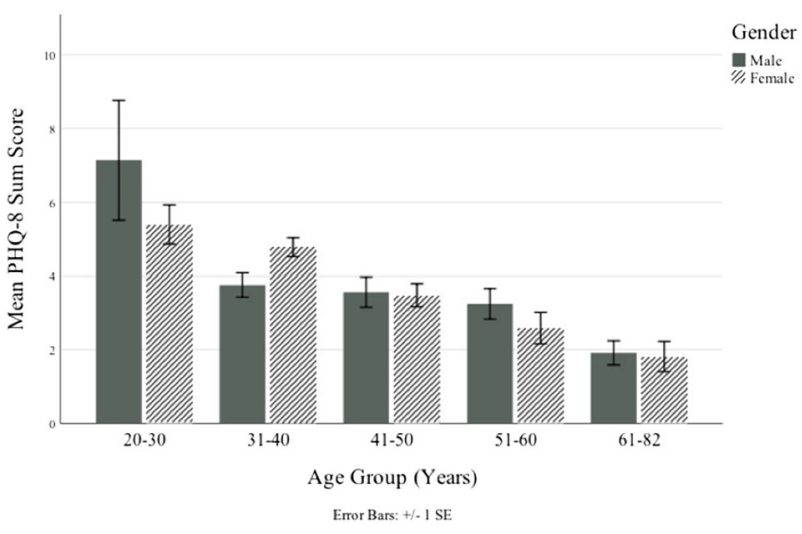

Fig. 2 Differences in mean PHQ-8 scores (raw scores) by parent age and gender

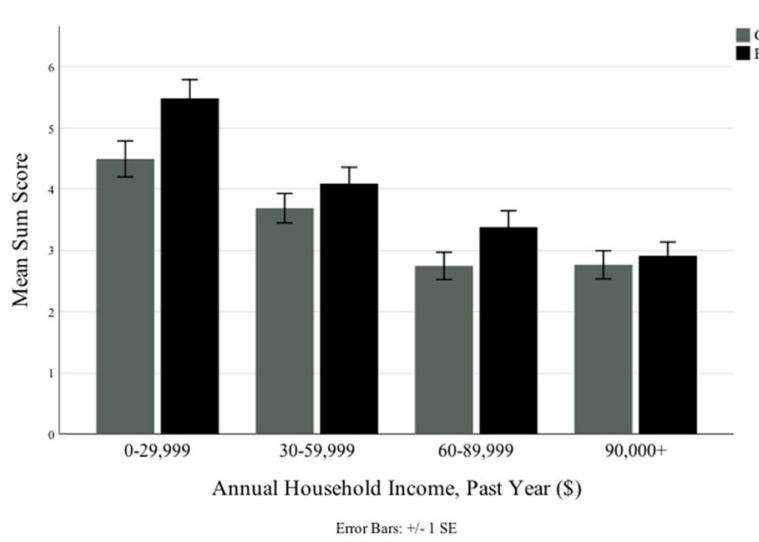

Fig. 3 Differences in mean GAD-7 and PHQ-8 scores (raw scores) by household income group

home (see Table 1); effect sizes were small across the board (i.e., Cohen's $d$ or $f \leq .2$ ). Relative to males, females reported higher levels of generalized anxiety symptoms $(t(1349)=-2.91, p=.004$, Cohen's $d=.15)$ and depressive symptoms $(t(1290)=-2.54, p=.011$, Cohen's $d$ $=.13$ ). An overall effect of age was found for both the GAD-7 $(F(4,1565)=14.18, p<.001$, Cohen's $f=.20)$ and PHQ-8 $(F(4,1565)=13.04, p<.001$, Cohen's $f=.19)$, with younger parents reporting higher symptoms. An effect of income group was also found for the GAD-7 $(F(3,1431)=$ $10.81, p<.001$, Cohen's $f=.15)$ and PHQ-8 $(F(3,1431=$ $16.97, p<.001$, Cohen's $f=.19$ ), with parents in lower income groups reporting higher symptoms. Finally, an effect of marital status was found for the GAD-7 $(F(2,1569)=4.77, p=.009$, Cohen's $f=.07)$ and PHQ-8 $(F(2,1569)=5.64, \quad p=.004$, Cohen's $f=.08)$, such that never married parents reported significantly higher symptoms of anxiety and depression relative to parents who were married or in civil or domestic partnerships. Results from Bonferroni post-hoc comparison tests can be found in Table 1.
Table 2 GAD-7 percentile scores for the total sample and by parent gender

\begin{tabular}{|c|c|c|c|}
\hline \multirow[b]{2}{*}{ Sum Score } & \multicolumn{3}{|l|}{ Percentiles $^{\mathrm{a}}$} \\
\hline & Total $(n=1570)$ & Males $(n=570)$ & Females $(n=1000)$ \\
\hline 0 & 21 & 21 & 20 \\
\hline 1 & 47 & 48 & 46 \\
\hline 2 & 58 & 60 & 56 \\
\hline 3 & 65 & 68 & 64 \\
\hline 4 & 71 & 74 & 69 \\
\hline 5 & 75 & 79 & 73 \\
\hline 6 & 79 & 83 & 77 \\
\hline 7 & 82 & 86 & 80 \\
\hline 8 & 85 & 87 & 83 \\
\hline 9 & 86 & 89 & 85 \\
\hline 10 & 88 & 91 & 87 \\
\hline 11 & 90 & 93 & 88 \\
\hline 12 & 91 & 94 & 90 \\
\hline 13 & 93 & 95 & 91 \\
\hline 14 & 94 & 96 & 93 \\
\hline 15 & 95 & 98 & 94 \\
\hline 16 & 96 & 98 & 96 \\
\hline 17 & 97 & 98 & 97 \\
\hline 18 & 98 & 99 & 98 \\
\hline 19 & 99 & 99 & 99 \\
\hline 20 & 99 & 100 & 99 \\
\hline 21 & 100 & 100 & 100 \\
\hline
\end{tabular}

The GAD-7 measures symptoms of generalized anxiety

${ }^{\text {a }}$ Percentiles indicate the rank of the subject compared to other subjects of the same group (i.e., gender)

\section{Clinically Significant Symptoms}

Percentages of parents with GAD-7 and PHQ-8 scores at or above 10 are depicted in Table 4. Moderate to severe symptoms of depression or generalized anxiety were reported by $17.7 \%$ of parents and $9.2 \%$ of parents reported moderate to severe symptoms of both depression and anxiety. Additionally, $12.7 \%$ of parents reported moderate to severe symptoms of generalized anxiety and $14.1 \%$ reported moderate to severe symptoms of depression. Though not depicted in the table, $3.6 \%$ of parents reported moderate to severe levels of generalized anxiety only (i.e., GAD-7 $\geq 10$ and PHQ- $8<10$ ), and $5.0 \%$ of parents reported moderate to severe levels of depression only (i.e., PHQ- $8 \geq$ 10 and GAD- $7<10$ ). Percentages of parents with GAD-7 or PHQ-8 scores at or above 10 differed significantly by gender $\left(\chi^{2}(1, N=1570)=5.42, p=.020\right)$, age group $\left(\chi^{2}(2\right.$, $N=1570)=33.03, p<.001)$, and income group $\left(\chi^{2}(2, N=\right.$ $1570)=39.74, p<.001)$. 
Table 3 PHQ-8 percentile scores for the total sample and by parent gender

\begin{tabular}{|c|c|c|c|}
\hline \multirow[b]{2}{*}{ Sum Score } & \multicolumn{3}{|l|}{ Percentiles $^{\mathrm{a}}$} \\
\hline & Total $(n=1570)$ & Males $(n=570)$ & Females $(n=1000)$ \\
\hline 0 & 18 & 19 & 18 \\
\hline 1 & 42 & 43 & 42 \\
\hline 2 & 52 & 54 & 51 \\
\hline 3 & 60 & 63 & 58 \\
\hline 4 & 65 & 69 & 63 \\
\hline 5 & 71 & 75 & 69 \\
\hline 6 & 76 & 80 & 73 \\
\hline 7 & 79 & 83 & 77 \\
\hline 8 & 82 & 86 & 80 \\
\hline 9 & 85 & 88 & 83 \\
\hline 10 & 87 & 90 & 85 \\
\hline 11 & 88 & 91 & 87 \\
\hline 12 & 90 & 92 & 88 \\
\hline 13 & 91 & 93 & 90 \\
\hline 14 & 92 & 94 & 92 \\
\hline 15 & 94 & 95 & 93 \\
\hline 16 & 95 & 96 & 95 \\
\hline 17 & 97 & 97 & 96 \\
\hline 18 & 97 & 98 & 97 \\
\hline 19 & 98 & 99 & 98 \\
\hline 20 & 99 & 99 & 99 \\
\hline 21 & 99 & 99 & 99 \\
\hline 22 & 99 & 99 & 99 \\
\hline 23 & 100 & 100 & 100 \\
\hline 24 & 100 & 100 & 100 \\
\hline
\end{tabular}

The PHQ-8 measures symptoms of depression

${ }^{\text {a}}$ Percentiles indicate the rank of the subject compared to other subjects of the same group (i.e., gender)

\section{Discussion}

The present study provides normative levels and demographic correlates of generalized anxiety and depressive symptoms in a national sample of parents living in the United States. All parents reported at least one child between the age of 5 and 12 years liviXung in the home. Consistent with hypotheses and prior reports (Brody et al., 2018; Kessler et al., 2005; Ertel et al., 2011), females and parents younger in age and with lower household incomes reported higher symptoms of anxiety and depression. Never married parents also reported significantly higher symptoms of anxiety and depression relative to currently married participants; this could be related to the findings that never married participants were more likely to be female, younger in age, and in the lowest income group. Racial/ethnic differences in mean levels of depressive and anxiety symptoms were not supported. Symptoms did not significantly differ by total number of children in the home, which is consistent with past research in mothers (Ertel et al., 2011).

Scores of 10 and above on the GAD-7 and PHQ- 8 are considered moderate to severe levels of generalized anxiety and depression, respectively, with an $80-100 \%$ sensitivity rate for an anxiety or depressive disorder per the DSM-IV or DSM-5 (e.g., Kroenke et al., 2001, 2009, 2010; Spitzer et al., 2006). In the present sample, participants reporting the lowest incomes $(<\$ 30,000)$ were over twice as likely to report moderate to severe symptoms of anxiety and depression relative to participants reporting incomes of $\$ 60,000$ and above. A similar result was seen for age; participants ages 20-40 years were over twice as likely to report moderate to severe symptoms of anxiety and depression relative to participants ages 51 and above. Unsurprisingly, GAD-7 and PHQ-8 scores were highly correlated, with $9.2 \%$ of the sample reporting moderate to severe symptoms of both generalized anxiety and depression and only 3-5\% of parents reporting moderate to severe symptom levels on only one questionnaire. Of note, however, we did not collect diagnostic data or data on functional impairment in this sample.

Normative symptom levels in this sample were higher than average PHQ-8 and GAD-7 scores found in the general population of adults, which was expected given that parents may experience unique stressors related to child-rearing and is consistent with prior research on rates of depression and anxiety in parents (e.g., England \& Sim, 2009; Ertel et al., 2011). For example, over $14 \%$ of parents in the present study reported scores of 10 and above on the PHQ-8. In comparison, Kroenke et al. (2009) found that $8.6 \%$ of a nationally representative sample of almost 200,000 adults living in the U.S. reported scores at or above 10 on the PHQ-8. Similarly, data collected from the National Health and Nutrition Examination Survey between 2013 and 2016 showed that $8.1 \%$ of American adults $(10.4 \%$ of women) reported PHQ-9 scores of 10 and above (Brody et al., 2018), and past-year MDD prevalence in adults is estimated around 8\% (Kessler et al., 2012). Based on a $100 \%$ sensitivity rate and $95 \%$ specificity rate for MDD using a PHQ-8 threshold of 10 (Kroenke et al., 2009), we may hypothesize that around $14 \%$ of parents in our sample would meet diagnostic criteria for MDD, which is similar to rates presented for mothers by Ertel et al. (2011).

In the original report on the GAD-7, over $20 \%$ of participants (2739 adults ages 18-95 recruited from primary care sites across 12 U.S. states) received scores of 10 or higher (Spitzer et al., 2006). However, rates of GAD in this report may have been higher than in the general population given the primary care setting. Indeed, Spitzer et al. (2006) reported that $9 \%$ of women and $4 \%$ of men in their sample met diagnostic criteria for GAD, which is higher than the 
Table 4 Percent of parents with GAD-7 and PHQ-8 total scores at or above levels suggesting clinically significant symptoms $(\geq 10)^{\mathrm{a}}$ by parent gender, age, and household income

\begin{tabular}{lllll}
\hline & GAD-7 $\geq 10$ & PHQ-8 $\geq 10$ & GAD-7 or PHQ-8 $\geq 10$ & GAD-7 \& PHQ-8 $\geq 10$ \\
\hline $\begin{array}{l}\text { Total Sample }-\% \\
\text { Gender }-\%\end{array}$ & 12.7 & 14.1 & 17.7 & 9.2 \\
Female $(n=1000)$ & 14.2 & 15.8 & 19.4 & 10.6 \\
Male $(n=570)$ & 10.2 & 11.2 & 14.7 & 6.7 \\
Age Group ${ }^{\mathrm{b}}-\%$ & & & \\
20-40 years $(n=848)$ & 17.3 & 17.5 & 22.6 & 12.1 \\
$41-50$ years $(n=398)$ & 8.3 & 11.8 & 13.8 & 6.3 \\
$51-82$ years $(n=324)$ & 6.2 & 8.3 & 9.6 & 4.9 \\
Income Group ${ }^{\mathrm{b}}-\%$ & & & & 14.2 \\
$\$ 0-\$ 29,999(n=372)$ & 19.1 & 22.3 & 27.2 & 10.0 \\
$\$ 30,000-\$ 59,999(n=418)$ & 15.3 & 14.6 & 19.9 & 6.0 \\
$\$ 60,000+(n=645)$ & 8.1 & 9.6 & 11.6 & \\
\hline
\end{tabular}

The GAD-7 measures symptoms of generalized anxiety and the PHQ-8 measures symptoms of depression; $\%=$ percent of subsample (e.g., \% of females with scores at or above 10)

${ }^{a}$ As determined by Kroenke et al. (2001) and Spitzer et al. (2006)

${ }^{\mathrm{b}}$ Age is presented in three groups, as participants ages 20-30 and 31-40 did not significantly differ in mean GAD-7 or PHQ-8 scores, nor did participants ages 51-60 and 61-82 (see Table 1). Similarly, we collapsed across income groups $\$ 60-89,999$ and $\$ 90,000+$, as these groups did not significantly differ in mean GAD-7 or PHQ-8 scores (see Table 1) estimated point prevalence around $2 \%$ or past-year prevalence around $3 \%$ for GAD in the general population (Wittchen et al., 1994; Kessler et al., 2005, 2012). In a study validating the GAD-7 in the general population of adults in Germany, approximately 5\% of adults had GAD-7 scores of 10 or greater (Löwe et al., 2008). Comparing the percentage of parents in the present study with GAD-7 scores of 10 or higher $(12.7 \%)$ to this latter study (Löwe et al., 2008), or to GAD rates estimated in the general population, could actually suggest more symptoms of generalized anxiety (and potentially higher corresponding rates of GAD) in parents relative to non-parents. An alternative explanation for differences between normative levels found in the present study and past research could be changes in symptoms over time, though research suggests that prevalence rates of anxiety disorders have not changed significantly over the years (Bandelow \& Michaelis, 2015) and that rates of depression around 2007 were not significantly different from rates around 2016 (Brody et al., 2018).

Additional limitations of the present study are worth noting. First, GAD is only one anxiety disorder; normative levels of other anxiety symptoms, such as social anxiety or panic symptoms, may also be important to consider in this population in future work. Second, this was a unique sample recruited for having at least one child between the ages of 5 and 12. Findings may not generalize to parents of younger children or older adolescents who may experience different challenges that pose differential risk for psychopathology. Third, the GAD-7 and PHQ-8 were originally normed using pen-and-paper methods, though these questionnaires have been validated for online distribution (e.g.,
Erbe et al., 2016; van Ballegooijen et al., 2016). It is possible that scores on these and similar measures could be higher or lower when administered online relative to when they are administered in person using pen-and-paper methods, though this is not consistently supported in the literature (e.g., Erbe et al., 2016; Alfonsson et al., 2014). Further, these questionnaires are frequently administered online in treatment and medical settings, as well as in research studies. Thus, the present norms for parents of young children are critical additions to the literature. Fourth, self-selection bias is a concern when conducting web panel surveys (Bethlehem, 2010; Keiding \& Louis, 2016); individuals who join web panel surveys are likely to differ from those who do not. In particular, it is possible that parents who did not respond to this survey differed from parents who did on levels of depression and/or anxiety. Parents higher in depressive symptoms may be less likely to complete the survey due to fatigue or low motivation. In addition, adults with less than a high school degree are less likely to use the internet (Anderson et al., 2019), and individuals who are worried about anonymity or do not trust technology may be less likely to respond to web-based surveys (Thompson et al., 2003), perhaps also biasing the present sample. Relatedly, though the sample was recruited based on estimates of population characteristics, these estimates will always have some error (Bethlehem, 2010). Indeed, though the sample was matched to the population (i.e., U.S. parents of children ages 5-12 years) on key variables such as race, ethnicity, income, and U.S. region, the sample included a higher percentage of female parents and guardians relative to male parents and 
guardians. This should be considered when interpreting results. Finally, these questionnaires are subject to limitations inherent in self-report measures (e.g., social acceptability bias), and, as mentioned, no formal diagnostic procedures were in place to verify symptoms or assess clinical significance or functional impairment.

Despite these limitations, this study is strengthened by the large, national sample and use of two widely used and validated questionnaires to measure generalized anxiety and depressive symptoms. Physicians, clinicians, and researchers may use the data presented in this report when treating or assessing anxiety and depression in U.S. parents. These data are particularly important now given worsening mental health reported by parents during the COVID-19 pandemic (Patrick et al., 2020), potentially related to dramatic changes in parents' routines and responsibilities (Lee et al., 2021), such as supporting their children in online or home schooling while trying to work from home. Isolation from friends and extended family and the widespread loss of jobs following the onset of the pandemic and continuing today may also contribute to increased symptoms of anxiety and depression in parents (Nicola et. al., 2020). The data presented in this study, which were collected pre-pandemic, provide important benchmarks that will be useful to estimate the impact of pandemic-related changes (e.g., school closures) on symptoms of depression and generalized anxiety in parents of school-age children.

Funding This study was supported by a grant to the last author [OL] from the Eunice Kennedy Shriver National Institute of Child Health and Human Development (R21HD090145).

\section{Compliance with Ethical Standards}

Conflict of Interest The authors declare no competing interests.

Consent to Participate Informed consent was obtained from all individual participants included in the study.

Ethics Approval This study was performed in line with the principles of the Declaration of Helsinki. Approval was granted by the Institutional Review Board at the University of Pittsburgh.

Publisher's note Springer Nature remains neutral with regard to jurisdictional claims in published maps and institutional affiliations.

\section{References}

Alfonsson, S., Maathz, P., \& Hursti, T. (2014). Interformat reliability of digital psychiatric self-report questionnaires: a systematic review. Journal of Medical Internet Research, 16(12), e268. https://doi.org/10.2196/jmir.3395

American Psychiatric Association (1994). Diagnostic and statistical manual of mental disorders (4th ed.). Washington, DC: American Psychiatric Association.
American Psychiatric Association (2013). Diagnostic and statistical manual of mental disorders (5th ed.). Washington, DC: American Psychiatric Association. https://doi.org/10.1176/appi.books. 9780890425596.

Anderson, M., Perrin, A., Jiang, J., \& Kumar, M. (2019, April 22). $10 \%$ of Americans don't use the internet. Who are they? https://www.pewresearch.org/fact-tank/2019/04/22/some-america ns-dont-use-the-internet-who-are-they/.

Asnaani, A., Richey, J. A., Dimaite, R., Hinton, D. E., \& Hofmann, S. G. (2010). A cross-ethnic comparison of lifetime prevalence rates of anxiety disorders. The Journal of Nervous and Mental Disease, 198(8), 551. https://doi.org/10.1097/NMD. 0b013e3181ea169f.

Bailey, R. K., Mokonogho, J. \& Kumar, A. (2019). Racial and ethnic differences in depression: current perspectives. Neuropsychiatric Disease \& Treatment, 15, 603-609. https://doi.org/10.2147/NDT. S128584.

Bandelow, B. \& Michaelis, S. (2015). Epidemiology of anxiety disorders in the 21st century. Dialogues in Clinical Neuroscience, 17(3), 327-335. PMID: 26487813.

Barker, E. D., Jaffee, S., Uher, R. \& Maughan, B. (2011). The contribution of prenatal and postnatal maternal anxiety and depression to child maladjustment. Depression \& Anxiety, 28(8), 696-702. https://doi.org/10.1192/bjp.bp.111.092346.

Bethlehem, J. (2010). Selection bias in web surveys. International Statistical Review, 78(2), 161-188. https://doi.org/10.1111/j. 1751-5823.2010.00112.x.

Breslau, J., Kendler, K. S., Su, M., Gaxiola-Aguilar, S. \& Kessler, R. C. (2005). Lifetime risk and persistence of psychiatric disorders across ethnic groups in the United States. Psychological Medicine, 35(3), 317-327. https://doi.org/10.1017/s0033291704003514.

Brody, D. J., Pratt, L. A., \& Hughes, J. P. (2018). Prevalence of depression among adults aged 20 and over: United States, 2013-2016. NCHS Data Brief, 303, 1-8. PMID: 29638213.

Bromet, E., Andrade, L. H., Hwang, I., Sampson, N. A., Alonso, J., De Girolamo, G. \& Karam, A. N. (2011). Cross-national epidemiology of DSM-IV major depressive episode. BMC Medicine, 9 (1), 90. https://doi.org/10.1186/1741-7015-9-90.

Cohen, J. (1988). The effect size index: d. Statistical Power Analysis Behavioral Sciences, 2(1), 284-288. https://doi.org/10.4324/ 9780203771587.

Crockett, L. J., Randall, B. A., Shen, Y. L., Russell, S. T. \& Driscoll, A. K. (2005). Measurement equivalence of the center for epidemiological studies depression scale for Latino and Anglo adolescents: a national study. Journal of Consulting \& Clinical Psychology, 73(1), 47-58. https://doi.org/10.1037/0022-006X.73. 1.47 .

Cuijpers, P., Vogelzangs, N., Twisk, J., Kleiboer, A., Li, J. \& Penninx, B. W. (2013). Differential mortality rates in major and subthreshold depression: Meta-analysis of studies that measured both. The British Journal Psychiatry, 202(1), 22-27. https://doi. org/10.1192/bjp.bp.112.112169.

England, M. J., Sim, L. J., \& National Research Council. (2009). Associations between depression in parents and parenting, child health, and child psychological functioning. Depression in Parents, Parenting, and Children: Opportunities to Improve Identification, Treatment, and Prevention. National Academies Press (US).

Erbe, D., Eichert, H. C., Rietz, C. \& Ebert, D. (2016). Interformat reliability of the patient health questionnaire: validation of the computerized version of the PHQ-9. Internet Interventions, 5, 1-4. https://doi.org/10.1016/j.invent.2016.06.006.

Ertel, K. A., Rich-Edwards, J. W. \& Koenen, K. C. (2011). Maternal depression in the United States: Nationally representative rates and risks. Journal Women's Health, 20(11), 1609-1617. https:// doi.org/10.1089/jwh.2010.2657. 
Garber, J. \& Cole, D. A. (2010). Intergenerational transmission of depression: A launch and grow model of change across adolescence. Development and Psychopathology, 22(4), 819-830. https://doi.org/10.1017/S0954579410000489.

Goodman, S. H., Rouse, M. H., Connell, A. M., Broth, M. R., Hall, C. M. \& Heyward, D. (2011). Maternal depression and child psychopathology: A meta-analytic review. Clinical Child and Family Psychology Review, 14(1), 1-27. https://doi.org/10.1007/s10567010-0080-1.

Grant, B. F., Goldstein, R. B., Chou, S. P., Huang, B., Stinson, F. S., Dawson, D. A. \& Ruan, W. J. (2009). Sociodemographic and psychopathologic predictors of first incidence of DSM-IV substance use, mood and anxiety disorders: Results from the Wave 2 National Epidemiologic Survey on Alcohol and Related Conditions. Molecular Psychiatry, 14(11), 1051-1066. https://doi.org/ 10.1038/mp.2008.41.

Grant, B. F., Hasin, D. S., Stinson, F. S., Dawson, D. A., Ruan, W. J., Goldstein, R. B. \& Huang, B. (2005). Prevalence, correlates, comorbidity, and comparative disability of DSM-IV generalized anxiety disorder in the USA: Results from the National Epidemiologic Survey on Alcohol and Related Conditions. Psychological Medicine, 35(12), 1747-1759. https://doi.org/10.1017/ S0033291705006069.

Haller, H., Cramer, H., Lauche, R., Gass, F. \& Dobos, G. J. (2014). The prevalence and burden of subthreshold generalized anxiety disorder: A systematic review. BMC Psychiatry, 14(1), 128. https://doi.org/10.1186/1471-244X-14-128.

Hasin, D. S., Sarvet, A. L., Meyers, J. L., Saha, T. D., Ruan, W. J., Stohl, M. \& Grant, B. F. (2018). Epidemiology of adult DSM-5 major depressive disorder and its specifiers in the United States. JAMA Psychiatry, 75(4), 336-346. https://doi.org/10.1001/jama psychiatry.2017.4602.

Hays, R. D., Liu, H. \& Kapteyn, A. (2015). Use of Internet panels to conduct surveys. Behavior Research Methods, 47(3), 685-690. https://doi.org/10.3758/s13428-015-0617-9.

Himle, J. A., Baser, R. E., Taylor, R. J., Campbell, R. D. \& Jackson, J. S. (2009). Anxiety disorders among African Americans, blacks of Caribbean descent, and non-Hispanic whites in the United States. Journal of Anxiety Disorders, 23(5), 578-590. https://doi.org/10. 1016/j.janxdis.2009.01.002.

Huang, F. Y., Chung, H., Kroenke, K. \& Spitzer, R. L. (2006). Racial and ethnic differences in the relationship between depression severity and functional status. Psychiatric Services, 57(4), 498-503. https://doi.org/10.1176/ps.2006.57.4.498.

Jackman S (2020). pscl: Classes and Methods for R Developed in the Political Science Computational Laboratory. United States Studies Centre, University of Sydney, Sydney, New South Wales, Australia. R package version 1.5.5, https://github.com/atahk/pscl/.

Kane, P. \& \& Garber, J. (2004). The relations among depression in fathers, children's psychopathology, and father-child conflict: A meta-analysis. Clinical Psychology Review, 24(3), 339-360. https://doi.org/10.1016/j.cpr.2004.03.004.

Keiding, N. \& Louis, T. A. (2016). Perils and potentials of selfselected entry to epidemiological studies and surveys. JR Stat Soc Ser A Stat Soc, 179(2), 319-376. https://doi.org/10.1111/rssa. 12136.

Kessler, R. C., Berglund, P., Demler, O., Jin, R., Koretz, D., Merikangas, K. R. \& Wang, P. S. (2003). The epidemiology of major depressive disorder: Results from the National Comorbidity Survey Replication (NCS-R). JAMA, 289(23), 3095-3105. https://doi.org/10.1001/jama.289.23.3095.

Kessler, R. C., Berglund, P., Demler, O., Jin, R., Merikangas, K. R. \& Walters, E. E. (2005). Lifetime prevalence and age-of-onset distributions of DSM-IV disorders in the National Comorbidity Survey Replication. Archives of General Psychiatry, 62(6), 593-602. https://doi.org/10.1001/archpsyc.62.6.593.
Kessler, R. C., Brandenburg, N., Lane, M., Roy-Byrne, P., Stang, P. D., Stein, D. J. \& Wittchen, H. U. (2005). Rethinking the duration requirement for generalized anxiety disorder: evidence from the National Comorbidity Survey Replication. Psychological Medicine, 35(7), 1073-1082. https://doi.org/10.1017/ s0033291705004538.

Kessler, R. C., Petukhova, M., Sampson, N. A., Zaslavsky, A. M. \& Wittchen, H. U. (2012). Twelve-month and lifetime prevalence and lifetime morbid risk of anxiety and mood disorders in the United States. International Journal of Methods in Psychiatric Research, 21(3), 169-184. https://doi.org/10.1002/mpr.1359.

Kroenke, K. \& Spitzer, R. L. (2002). The PHQ-9: A new depression diagnostic and severity measure. Psychiatric Annals, 32(9), 509-515. https://doi.org/10.3928/0048-5713-20020901-06.

Kroenke, K., Spitzer, R. L. \& Williams, J. B. (2001). The PHQ-9: Validity of a brief depression severity measure. Journal of General Internal Medicine, 16(9), 606-613. https://doi.org/10.1046/j. 1525-1497.2001.016009606.x.

Kroenke, K., Spitzer, R. L., Williams, J. B. \& Löwe, B. (2010). The patient health questionnaire somatic, anxiety, and depressive symptom scales: A systematic review. General Hospital Psychiatry, 32(4), 345-359. https://doi.org/10.1016/j.genhosppsych. 2010.03.006.

Kroenke, K., Strine, T. W., Spitzer, R. L., Williams, J. B., Berry, J. T. \& Mokdad, A. H. (2009). The PHQ-8 as a measure of current depression in the general population. Journal of Affective Disorders, 114(1-3), 163-173. https://doi.org/10.1016/j.jad.2008.06. 026.

Lawrence, P. J., Murayama, K. \& Creswell, C. (2019). Systematic review and meta-analysis: Anxiety and depressive disorders in offspring of parents with anxiety disorders. Journal of the American Academy of Child \& Adolescent Psychiatry, 58(1), 46-60. https://doi.org/10.1016/j.jaac.2018.07.898.

Lee, P. W., Schulberg, H. C., Raue, P. J. \& Kroenke, K. (2007). Concordance between the PHQ-9 and the HSCL-20 in depressed primary care patients. Journal of Affective Disorders, 99(1-3), 139-145. https://doi.org/10.1016/j.jad.2006.09.002.

Lee, S., Ward, K., Chang, O. \& Downing, K. (2021). Parenting activities and the transition to home-based education during the COVID-19 pandemic. Children and Youth Services Review, 122, 105585. https://doi.org/10.1016/j.childyouth.2020.105585.

Lieb, R., Isensee, B., Höfler, M., Pfister, H. \& Wittchen, H. U. (2002). Parental major depression and the risk of depression and other mental disorders in offspring: A prospective-longitudinal community study. Archives of General Psychiatry, 59(4), 365-374. https://doi.org/10.1001/archpsyc.59.4.365.

Lindhiem, O., Vaughn-Coaxum, R. A., Higa, J., Harris, J. L., Kolko, D. J. \& Pilkonis, P. A. (2019). Development and validation of the Knowledge of Effective Parenting Test (KEPT) in a nationally representative sample. Psychological Assessment, 31(6), 781-792. https://doi.org/10.1037/pas0000699.

Lovejoy, M. C., Graczyk, P. A., O'Hare, E. \& Neuman, G. (2000). Maternal depression and parenting behavior: A meta-analytic review. Clinical Psychology Review, 20(5), 561-592. https://doi. org/10.1016/s0272-7358(98)00100-7.

Löwe, B., Spitzer, R. L., Williams, J. B., Mussell, M., Schellberg, D. \& Kroenke, K. (2008). Depression, anxiety and somatization in primary care: Syndrome overlap and functional impairment. General Hospital Psychiatry, 30(3), 191-199. https://doi.org/10. 1016/j.genhosppsych.2008.01.001.

Mughal, M. K., Giallo, R., Arnold, P., Benzies, K., Kehler, H., Bright, K. \& Kingston, D. (2018). Trajectories of maternal stress and anxiety from pregnancy to three years and child development at 3 years of age: Findings from the All Our Families (AOF) pregnancy cohort. Journal of Affective Disorders, 234, 318-326. https://doi.org/10.1016/j.jad.2018.02.095. 
Nicola, M., Alsafi, Z., Sohrabi, C., Kerwan, A., Al-Jabir, A., Iosifidis, C. \& Agha, R. (2020). The socio-economic implications of the coronavirus and COVID-19 pandemic: A review. International Journal of Surgery, 78, 185-193. https://doi.org/10.1016/j.ijsu. 2020.04.018.

Patrick, S. W., Henkhaus, L. E., Zickafoose, J. S., Lovell, K., Halvorson, A., Loch, S.,... \& Davis, M. M. (2020). Well-being of parents and children during the COVID-19 pandemic: A national survey. Pediatrics, 146(4), e2020016824.

Perreira, K. M., Deeb-Sossa, N., Harris, K. M. \& Bollen, K. (2005). What are we measuring? An evaluation of the CES-D across race/ ethnicity and immigrant generation. Social Forces, 83, 1567-1601. https://doi.org/10.1353/sof.2005.0077.

Pew Research Center (2019). Internet/Broadband Fact Sheet. https://www.pewresearch.org/internet/fact-sheet/internet-broa dband/.

Pine, D. S., Cohen, P., Gurley, D., Brook, J. \& Ma, Y. (1998). The risk for early-adulthood anxiety and depressive disorders in adolescents with anxiety and depressive disorders. Archives of General Psychiatry, 55(1), 56-64. https://doi.org/10.1001/a rchpsyc.55.1.56.

Ramchandani, P. G., Stein, A., O’Connor, T. G., Heron, J. O. N., Murray, L. \& Evans, J. (2008). Depression in men in the postnatal period and later child psychopathology: A population cohort study. Journal American Academy Child \& Adolescent Psychiatry, 47(4), 390-398. https://doi.org/10.1097/CHI.0b013e31816429c2.

Riolo, S. A., Nguyen, T. A., Greden, J. F. \& King, C. A. (2005). Prevalence of depression by race/ethnicity: Findings from the National Health and Nutrition Examination Survey III. American Journal of Public Health, 95(6), 998-1000. https://doi.org/10. 2105/AJPH.2004.047225.

Rucci, P., Gherardi, S., Tansella, M., Piccinelli, M., Berardi, D., Bisoffi, G. \& Pini, S. (2003). Subthreshold psychiatric disorders in primary care: Prevalence and associated characteristics. Journal of Affective Disorders, 76(1-3), 171-181. https://doi.org/10. 1016/s0165-0327(02)00087-3.

Salk, R. H., Hyde, J. S. \& Abramson, L. Y. (2017). Gender differences in depression in representative national samples: Meta-analyses of diagnoses and symptoms. Psychological Bulletin, 143(8), 783-822. https://doi.org/10.1037/bul0000102.

Sequeira, S. L., Silk, J. S., Woods, W. C., Kolko, D. J. \& Lindhiem, O. (2019). Psychometric properties of the SCARED in a nationally representative US sample of 5-12-year-olds. Journal Clinical Child \& Adolescent Psychology, 1-12. https://doi.org/10.1080/ 15374416.2019.1614001.

Simon, G. E., Goldberg, D., Tiemens, B. G. \& Ustun, T. B. (1999). Outcomes of recognized and unrecognized depression in an international primary care study. General Hospital Psychiatry, 21 (2), 97-105. https://doi.org/10.1016/s0163-8343(98)00072-3.

Skipstein, A., Janson, H., Stoolmiller, M. \& Mathiesen, K. S. (2010). Trajectories of maternal symptoms of anxiety and depression. A 13-year longitudinal study of a population-based sample. BMC Public Health, 10(1), 1-11. https://doi.org/10.1186/1471-245810-589.

Spitzer, R. L., Kroenke, K., Williams, J. B. \& Löwe, B. (2006). A brief measure for assessing generalized anxiety disorder: The GAD-7. Archives Internal Medicine, 166(10), 1092-1097. https://doi.org/ 10.1001/archinte.166.10.1092.

Teetsel, R. N., Ginsburg, G. S. \& Drake, K. L. (2014). Anxietypromoting parenting behaviors: A comparison of anxious mothers and fathers. Child Psychiatry \& Human Development, 45(2), 133-142. https://doi.org/10.1007/s10578-013-0384-8.

Thompson, L. F., Surface, E. A., Martin, D. L. \& Sanders, M. G. (2003). From paper to pixels: Moving personnel surveys to the Web. Personnel Psychology, 56(1), 197-227. https://doi.org/10. 1111/j.1744-6570.2003.tb00149.x.

van Ballegooijen, W., Riper, H., Cuijpers, P., van Oppen, P. \& Smit, J. H. (2016). Validation of online psychometric instruments for common mental health disorders: a systematic review. BMC Psychiatry, 16(1), 1-12. https://doi.org/10.1186/s12888-0160735-7.

Vesga-Lфpez, O., Schneier, F., Wang, S., Heimberg, R., Liu, S. M., Hasin, D. S. \& Blanco, C. (2008). Gender differences in generalized anxiety disorder: Results from the National Epidemiologic Survey on Alcohol and Related Conditions (NESARC). The Journal Clinical Psychiatry, 69(10), 1606-1616. PMID: 19192444.

Van Der Waerden, J., Gal,ra, C., Larroque, B., Saurel-Cubizolles, M. J., Sutter-Dallay, A. L. \& Melchior, M. (2015). EDEN Mother-Child Cohort Study Group. (2015). Maternal depression trajectories and children's behavior at age 5 years. The Journal Pediatrics, 166, 1440-1448. https://doi.org/10.1016/j.jpeds.2015. 03.002.

WittchenH. U., ZhaoS., KesslerR. C. \& EatonW. W. (1994). DSM-III$\mathrm{R}$ generalized anxiety disorder in the National Comorbidity Survey. Archives of General Psychiatry, 51, 355-364. https://doi. org/10.1001/archpsyc.1994.03950050015002.

$\mathrm{Xu}, \mathrm{T}$., Zhu, G., \& Han, S. (2017). Study of depression influencing factors with zero-inflated regression models in a large-scale population survey. BMJ Open, 7(11), e016471. https://doi.org/10. 1136/bmjopen-2017-016471.

Zeileis, A., Kleiber, C., Jackman, S. (2008). Regression Models for Count Data in R. Journal of Statistical Software, 27(8). http://www.jstatsoft.org/v27/i08/. 